\title{
Roland Pulch
}

\section{Model order reduction in uncertainty quantification}

\begin{abstract}
Mathematical models include parameters, which are often affected by uncertainties due to measurement errors or imperfections of an industrial production, for example. In uncertainty quantification (UQ), parameter variations are often described by random variables or random processes. Of course the resulting stochastic model exhibits a higher complexity in comparison to the original model. Thus methods of model order reduction (MOR) become attractive to save computational effort in UQ. We consider dynamical systems consisting of ordinary differential equations or differential algebraic equations. The focus is on linear dynamical systems. On the one hand, state variables and output variables can be expanded into a series with given orthogonal polynomials and unknown coefficient functions. A stochastic Galerkin method yields a high-dimensional deterministic system satisfied by an approximation of the coefficient functions. A stochastic collocation method can also be written as a large weakly coupled deterministic system. We use traditional MOR methods to shrink the dimensionality of the large systems. On the other hand, quantities of interest typically represent probabilistic integrals like moments or failure probabilities, for example. Multidimensional quadrature methods and sampling techniques directly generate approximations of these statistics. Therein, the original dynamical system has to be solved many times for different realizations of the parameters. Thus high-dimensional dynamical systems cause a huge total computational effort. We discuss methods of parametric MOR to reuse a reduced-order model for different parameter values. Finally, numerical results are demonstrated for test examples, where we perform the reduction of large deterministic systems as well as parametric MOR.
\end{abstract}

Keywords: Model order reduction, uncertainty quantification, polynomial chaos, quadrature, reduced basis method

MSC 2010: 34C20, 37M99, 65D32, 65L99, 93A15

\subsection{Introduction}

In science and engineering, mathematical modeling often yields systems of ordinary differential equations (ODEs), differential algebraic equations (DAEs), or partial differential equations (PDEs). The systems include physical parameters or geometrical parameters, which exhibit uncertainties due to modeling errors, measurement errors, or

Roland Pulch, Universität Greifswald, Greifswald, Germany

¿ Open Access. ( 2021 Roland Pulch, published by De Gruyter. (cc)BY-NC-ND This work is licensed under the Creative Commons Attribution-NonCommercial-NoDerivatives 4.0 International License. 
imperfections of an industrial production. Uncertainty quantification (UQ) determines the sensitivity of the model outputs with respect to these parameter variations. Often a stochastic modeling is used, where uncertain parameters are replaced by random variables, random processes, or spatial random fields; see [44, 46]. Now the model output also becomes a random process or a random field.

The dynamical systems may be low-dimensional or high-dimensional. In any case, the presence of random variables increases the complexity of the differential equations. In some numerical methods, a stochastic discretization yields a deterministic dynamical system of a much higher dimension. Now methods of model order reduction (MOR) are attractive to decrease the complexity and thus save computational effort in the numerical simulation. Efficient MOR methods are already available for deterministic systems of differential equations or differential algebraic equations; see $[2,6,7,9,16,40]$.

We consider a polynomial chaos expansion (see [3, 45]) of the random quantity of interest (QoI) in a small- or medium-sized linear dynamical system. The expansion includes orthogonal basis polynomials and unknown time-dependent coefficient functions. The stochastic Galerkin method yields a coupled deterministic linear dynamical system of high dimensionality, whose solution approximates the coefficient functions; see [21, 31, 42]. MOR methods have been applied to this high-dimensional system in [15, 35, 36, 38], for example. Alternatively, a stochastic collocation technique using a quadrature rule or a sampling method provides approximations of the coefficient functions. We write the stochastic collocation method in the form of a weakly coupled deterministic linear dynamical system; see [33, 34]. Now the system is highdimensional and thus common MOR methods can be applied.

The concept of parametric MOR (pMOR) becomes attractive in the case of parameter-dependent systems with high dimensionality. Its aim is the efficient computation of reduced-order models (ROMs) for a (possibly) large number of realizations of the parameters. Methods of pMOR and their applications are demonstrated in $[5,8,13]$, for example. We address the usage of pMOR in problems of UQ. An ROM represents a surrogate model, which can be solved instead of the original dynamical system. Therein, statistics of the random QoI are computed like the moments, for example. We also discuss reduced basis methods, which can be seen as a class of pMOR methods. Reduced basis techniques are efficient for many spatial problems modeled by PDEs; see $[18,22,30]$. A rigorous investigation of reduced basis methods for UQ can be found in [11].

This chapter is organized as follows. We introduce UQ of dynamical systems and the stochastic modeling in Section 10.2. MOR of deterministic dynamical systems, which are generated by stochastic discretizations, are addressed in Section 10.3. PMOR of dynamical systems with random parameters is considered in Section 10.4. Finally, we illustrate numerical simulations of test examples for both cases in Section 10.5. 


\subsection{Stochastic models and methods}

We review the stochastic modeling and numerical techniques to solve the arising problems in this section.

\subsubsection{Dynamical systems}

Let a nonlinear dynamical system be given in the form

$$
\begin{aligned}
E(\boldsymbol{\mu}) \dot{\mathbf{x}}(t, \boldsymbol{\mu}) & =A(\boldsymbol{\mu}) \mathbf{x}(t, \boldsymbol{\mu})+\mathbf{F}(\mathbf{x}(t, \boldsymbol{\mu}), \boldsymbol{\mu})+B(\boldsymbol{\mu}) \mathbf{u}(t), \\
\mathbf{y}(t, \boldsymbol{\mu}) & =C(\boldsymbol{\mu}) \mathbf{x}(t, \boldsymbol{\mu}),
\end{aligned}
$$

with matrices and functions depending on physical and/or geometrical parameters $\boldsymbol{\mu} \in \mathcal{D} \subseteq \mathbb{R}^{p}$. The sizes of the matrices are $A, E \in \mathbb{R}^{n \times n}, B \in \mathbb{R}^{n \times n_{\text {in }}}, C \in \mathbb{R}^{n_{\text {out }} \times n}$. The system involves a nonlinear function $\mathbf{F}: \mathbb{R}^{n} \times \mathcal{D} \rightarrow \mathbb{R}^{n}$. For nonsingular matrices $E$, the system consists of ODEs with the state variables $\mathbf{x}:\left[t_{0}, T\right] \times \mathcal{D} \rightarrow \mathbb{R}^{n}$. For singular matrices $E$, a system of DAEs is given with the inner variables $\mathbf{x}$. We consider initial value problems

$$
\mathbf{x}\left(t_{0}, \boldsymbol{\mu}\right)=\mathbf{x}_{0}(\boldsymbol{\mu}) \quad \text { for } \boldsymbol{\mu} \in \mathcal{D}
$$

with a predetermined function $\mathbf{x}_{0}: \mathcal{D} \rightarrow \mathbb{R}^{n}$. In the case of DAEs, the initial values have to satisfy consistency conditions and typically depend on the physical parameters of the system.

An input $\mathbf{u}:\left[t_{0}, T\right] \rightarrow \mathbb{R}^{n_{\text {in }}}$ is supplied to the system (10.1). An output $\mathbf{y}:\left[t_{0}, T\right] \times$ $\mathcal{D} \rightarrow \mathbb{R}^{n_{\text {out }}}$ is defined as a QoI by the state variables or inner variables and the matrix $C$. Without loss of generality, we restrict the analysis to the case of a single output, i. e., $n_{\text {out }}=1$.

Efficient methods of MOR are available for linear time-invariant dynamical systems of the form

$$
\begin{aligned}
E(\boldsymbol{\mu}) \dot{\mathbf{x}}(t, \boldsymbol{\mu}) & =A(\boldsymbol{\mu}) \mathbf{x}(t, \boldsymbol{\mu})+B(\boldsymbol{\mu}) \mathbf{u}(t) \\
y(t, \boldsymbol{\mu}) & =C(\boldsymbol{\mu}) \mathbf{x}(t, \boldsymbol{\mu})
\end{aligned}
$$

Typical MOR methods are balanced truncation, as described in Chapter 2 of Volume 1 of Model order reduction, and Krylov subspace methods, as described in Chapter 3 of Volume 1 of Model order reduction. Thus we focus on linear dynamical systems (10.3). We assume that the linear dynamical system (10.3) is asymptotically stable for all $\boldsymbol{\mu} \in \mathcal{D}$. Hence each eigenvalue $\lambda$ satisfying $\operatorname{det}(\lambda E(\boldsymbol{\mu})-A(\boldsymbol{\mu}))=0$ has a strictly negative real part.

In MOR, a dynamical system of a much lower dimension $r \ll n$ is constructed, whose output $\bar{y}$ is still a good approximation of the QoI $y$ in the original system (10.1) 
or (10.3). Let a fixed parameter value $\boldsymbol{\mu} \in \mathcal{D}$ be given. The ROM of the linear dynamical system (10.3) reads as

$$
\begin{aligned}
\bar{E}(\boldsymbol{\mu}) \dot{\overline{\mathbf{x}}}(t, \boldsymbol{\mu}) & =\bar{A}(\boldsymbol{\mu}) \mathbf{x}(t, \boldsymbol{\mu})+\bar{B}(\boldsymbol{\mu}) \mathbf{u}(t), \\
\bar{y}(t, \boldsymbol{\mu}) & =\bar{C}(\boldsymbol{\mu}) \overline{\mathbf{x}}(t, \boldsymbol{\mu}) .
\end{aligned}
$$

Projection-based MOR applies two matrices $V, W \in \mathbb{R}^{n \times r}$ of full rank. Typically, an orthogonal matrix $V$ is supposed, i.e., $V^{\top} V=I_{r}$ with identity matrix $I_{r} \in \mathbb{R}^{r \times r}$. The matrices of the linear dynamical system are reduced by

$$
\begin{array}{llrl}
\bar{A}(\boldsymbol{\mu}) & =W^{\top} A(\boldsymbol{\mu}) V, & \bar{B}(\boldsymbol{\mu})=W^{\top} B(\boldsymbol{\mu}), \\
\bar{C}(\boldsymbol{\mu})=C(\boldsymbol{\mu}) V, & \bar{E}(\boldsymbol{\mu})=W^{\top} E(\boldsymbol{\mu}) V .
\end{array}
$$

If the two projection matrices coincide $(V=W)$, then the MOR method is of Galerkin type. In common MOR, the projection matrices are computed for each required parameter value $\boldsymbol{\mu} \in \mathcal{D}$ separately. pMOR addresses the parameter variation in a whole domain $\mathcal{D}$, which is considered in Section 10.4.1.

MOR of general nonlinear dynamical systems (10.1) represents a critical task. More efficient MOR methods are available for quadratic-bilinear (QB) systems; see [1, 4] and Chapter 3 of Volume 1 of Model order reduction. Sometimes a nonlinear dynamical system can be transformed into an equivalent QB system. Alternatively, the construction of approximative QB systems is feasible.

Furthermore, an overview on software of MOR methods can be found in Chapter 13 of this volume.

\subsubsection{Stochastic modeling}

The parameters are often affected by uncertainties in the systems (10.1) or (10.3). A common approach in UQ is to consider the parameters as independent random variables $\boldsymbol{\mu}: \Omega \rightarrow \mathcal{D}$ on some probability space $(\Omega, \mathcal{A}, P)$ with event space $\Omega$, sigmaalgebra $\mathcal{A}$, and probability measure $P$. Often traditional probability distributions are applied like uniform, beta, Gaussian, etc. We assume that a joint probability density function $\rho: \mathcal{D} \rightarrow \mathbb{R}$ is available. Consequently, the output becomes a random process. The expected value of a measurable function $f: \mathcal{D} \rightarrow \mathbb{R}$ depending on the random variables reads as

$$
\mathbb{E}[f]=\int_{\Omega} f(\boldsymbol{\mu}(\omega)) \mathrm{d} P(\omega)=\int_{\mathcal{D}} f(\boldsymbol{\mu}) \rho(\boldsymbol{\mu}) \mathrm{d} \boldsymbol{\mu},
$$

provided that the integral exists. The moments are $\mathbb{E}\left[f^{k}\right]$ for positive integers $k$. The variance is the second central moment

$$
\operatorname{Var}[f]=\mathbb{E}\left[f^{2}\right]-\mathbb{E}[f]^{2}
$$


as usual and the standard deviation is its square root $\sigma[f]=\sqrt{\operatorname{Var}[f]}$. The skewness and the kurtosis also represent interesting statistical quantities. They are given by the third and fourth standardized moments, respectively, i.e.,

$$
\eta_{j}=\frac{\mathbb{E}\left[(f-\mathbb{E}[f])^{j}\right]}{\sigma[f]^{j}}
$$

for $j=3,4$.

In the dynamical systems (10.1) and (10.3), both the state variables and the output (QoI) change into random processes due to the stochastic modeling. Thus the complexity of the problem increases significantly.

\subsubsection{Quadrature and sampling}

In a stochastic model, the desired data typically represent probabilistic integrals of the form (10.6). For example, moments are defined by the powers of a function and failure probabilities are given by an indicator function or a characteristic function; see [19]. Since the QoI is the random process $y$, the integrands are time-dependent. Sometimes just the QoI at a final time $t=T$ is considered.

A well-known approach to discretize a probabilistic integral is a multivariate quadrature rule or a sampling method. Each technique is determined by a set of nodes $\left\{\boldsymbol{\mu}^{(1)}, \ldots, \boldsymbol{\mu}^{(k)}\right\} \subset \mathcal{D}$ in the parameter domain and a set of weights $\left\{\gamma_{1}, \ldots, \gamma_{k}\right\} \subset \mathbb{R}$. The sum of the weights is always one. The numerical approximation of a probabilistic integral becomes a finite sum

$$
\int_{\mathcal{D}} f(\boldsymbol{\mu}) \rho(\boldsymbol{\mu}) \mathrm{d} \boldsymbol{\mu} \approx \sum_{\ell=1}^{k} \gamma_{\ell} f\left(\boldsymbol{\mu}^{(\ell)}\right) .
$$

In the case of low numbers of random parameters (say, $p \leq 5$ ), we can use tensor product formulas of univariate quadrature rules. These methods become inefficient for higher dimensions due to the curse of dimensionality. Sparse grids or specific quadrature rules are available for large dimensions; see [17, 29, 43]. The curse of dimensionality is omitted in the sparse grid construction. A drawback is that often negative weights occur. However, also sparse grids become computationally infeasible for very high dimensions (say, $p>30$ ). Consequently, we have to apply sampling schemes like Monte Carlo or quasi-Monte Carlo methods; see [28]. The weights become $\gamma_{\ell}=\frac{1}{k}$ for all $\ell$ in each sampling scheme. Pseudo-random numbers or sequences of low discrepancy yield the nodes in a Monte Carlo or quasi-Monte Carlo method, respectively. Any number $k$ can be chosen in a sampling method. Yet high-dimensional problems require typically a large number $k$ to achieve sufficiently accurate results.

Figure 10.1 illustrates examples of the nodes for different methods in the case of two independent uniformly distributed random variables $\mu_{i} \in[0,1]$ for $i=1,2$. 
(i)

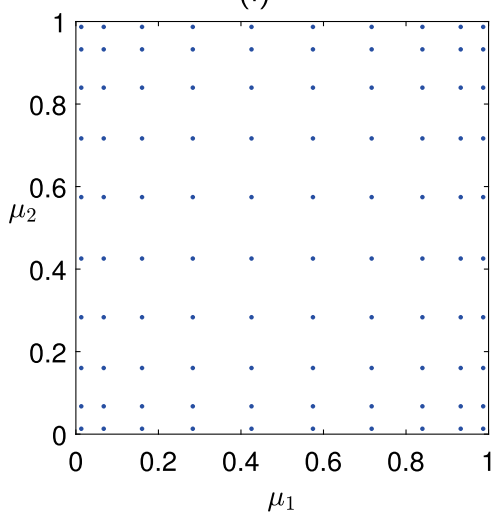

(iii)

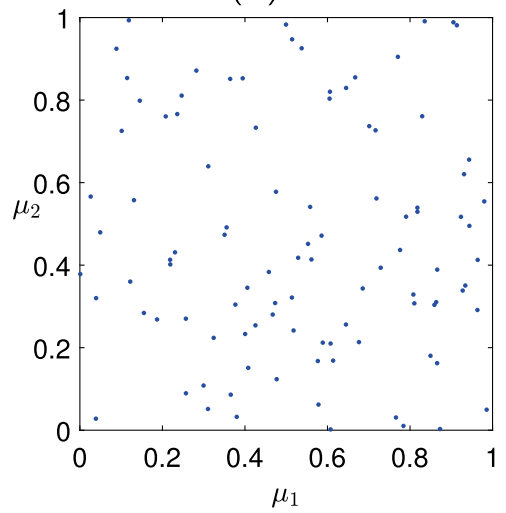

(ii)

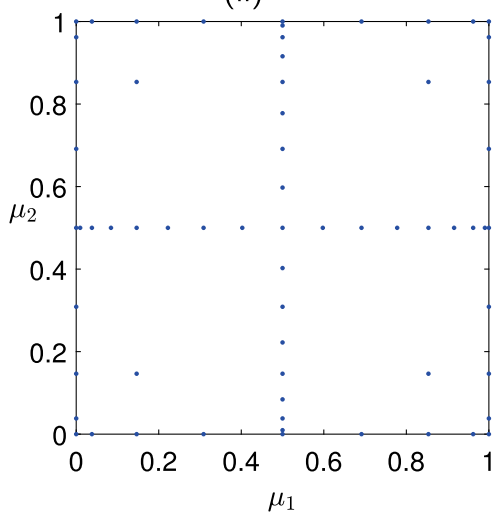

(iv)

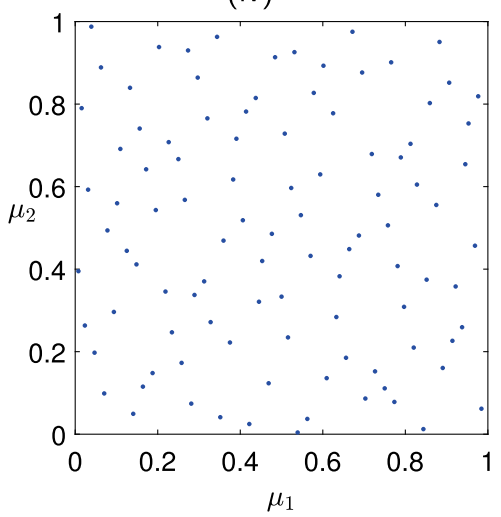

Figure 10.1: Nodes in quadrature rules or sampling methods for two uniformly distributed random variables: (i) tensor product Gauss-Legendre quadrature (100 points), (ii) sparse grid of level 4 based on the Clenshaw-Curtis rule (65 points), (iii) Monte Carlo with pseudo-random numbers (100 points), (iv) quasi-Monte Carlo with Halton sequence (100 points).

Since we consider dynamical systems (10.1) or (10.3) with a QoI, a function $f$ depends on $y$ in the integrand of (10.6), i. e., $f(\boldsymbol{\mu})=\tilde{f}(y(t, \boldsymbol{\mu}))$ for fixed $t$. Hence the evaluation of an approximation (10.9) requires to solve $k$ times an initial value problem of the dynamical system. This effort dominates the computation work in the stochastic model. The total effort is roughly proportional to $k$.

\subsubsection{Polynomial expansions}

The expected value (10.6) implies the inner product

$$
\langle f, g\rangle=\mathbb{E}[f g]=\int_{\mathcal{D}} f(\boldsymbol{\mu}) g(\boldsymbol{\mu}) \rho(\boldsymbol{\mu}) \mathrm{d} \boldsymbol{\mu}
$$


for two measurable functions depending on the random parameters. The associated Hilbert space is the set of square integrable functions

$$
\mathcal{L}^{2}(\mathcal{D}, \rho)=\left\{f: \mathcal{D} \rightarrow \mathbb{R}: f \text { measurable and } \mathbb{E}\left[f^{2}\right]<\infty\right\}
$$

Its norm reads as $\|f\|_{\mathcal{L}^{2}}=\sqrt{\langle f, f\rangle}$.

We apply an expansion of the random process $y$ into a set of orthogonal polynomials. Each traditional probability distribution exhibits a sequence of orthogonal polynomials (see [45]): Legendre polynomials for uniform distribution, Hermite polynomials for Gaussian distribution, Jacobi polynomials for beta distribution, etc. Let $\left(\phi_{j}^{(q)}\left(\mu_{q}\right)\right)_{j \in \mathbb{N}_{0}}$ be the sequence of univariate orthonormal polynomials associated to the $q$-th random variable. The degree of the $j$-th polynomial is exactly $j$. We assume that the orthonormal basis is complete, which holds true for Gaussian, uniform, beta, and other distributions. However, there are exceptions; see [12]. The multivariate polynomials are just the products of the univariate polynomials. The set of all basis polynomials up to total degree $d$ reads as

$$
\left\{\Phi_{i}(\boldsymbol{\mu})=\phi_{j_{1}}^{(1)}\left(\mu_{1}\right) \phi_{j_{2}}^{(2)}\left(\mu_{2}\right) \cdots \phi_{j_{p}}^{(p)}\left(\mu_{p}\right): j_{1}+j_{2}+\cdots+j_{p} \leq d\right\}
$$

There is a one-to-one mapping between the indices $i=1,2,3, \ldots$ and the multiindices $\left(j_{1}, j_{2}, \ldots, j_{p}\right)$. The basis polynomials $\left(\Phi_{i}\right)_{i \in \mathbb{N}}$ satisfy the orthogonality property

$$
\left\langle\Phi_{i}, \Phi_{k}\right\rangle= \begin{cases}0 & \text { for } i \neq k \\ 1 & \text { for } i=k\end{cases}
$$

with the inner product (10.10). The cardinality of the set (10.12) is (see [46])

$$
\left(\begin{array}{c}
p+d \\
d
\end{array}\right)=\frac{(p+d) !}{p ! d !}
$$

Hence the number of basis polynomials becomes large for large numbers $p$ of random variables, even if the total degree is moderate, say, $2 \leq d \leq 5$. Figure 10.2 illustrates the growth of the number of multivariate basis polynomials.

Series including the orthogonal basis functions are called polynomial chaos (PC) expansions. The technique is analogous to Fourier series, where the trigonometric polynomials are just replaced by the orthonormal polynomials (10.12) with respect to the inner product (10.10). We expand the QoI satisfying the linear dynamical system (10.3) into

$$
y(t, \boldsymbol{\mu})=\sum_{i=1}^{\infty} w_{i}(t) \Phi_{i}(\boldsymbol{\mu})
$$

with a priori unknown coefficient functions $w_{i}:\left[t_{0}, T\right] \rightarrow \mathbb{R}$ satisfying

$$
w_{i}(t)=\left\langle y(t, \boldsymbol{\mu}), \Phi_{i}(\boldsymbol{\mu})\right\rangle=\int_{\mathcal{D}} y(t, \boldsymbol{\mu}) \Phi_{i}(\boldsymbol{\mu}) \rho(\boldsymbol{\mu}) \mathrm{d} \boldsymbol{\mu}
$$




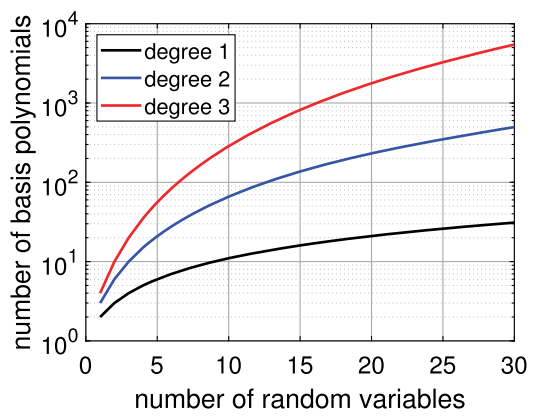

Figure 10.2: Number of basis polynomials in dependence on number of random variables for different total degrees (in semi-logarithmic scale).

for $i \in \mathbb{N}$. We obtain a finite approximation by a truncation of the series (10.14), i. e.,

$$
y^{(m)}(t, \boldsymbol{\mu})=\sum_{i=1}^{m} w_{i}(t) \Phi_{i}(\boldsymbol{\mu}) .
$$

The approximations (10.16) converge pointwise in time to the random process $y$ for $m \rightarrow \infty$ in the $\mathcal{L}^{2}$-norm provided that the basis is complete.

We include all basis polynomials (10.12) up to some total degree $d$, where the number $m$ is equal to (10.13). Let $\Phi_{1} \equiv 1$ be the constant polynomial of degree zero. The approximation (10.16) also yields the expected value as well as an approximation of the variance (10.7) via

$$
\mathbb{E}[y(t, \cdot)]=w_{1}(t) \text { and } \operatorname{Var}[y(t, \cdot)] \approx \sum_{i=2}^{m} w_{i}(t)^{2} .
$$

The task is to compute numerically the coefficient functions of the truncated series (10.16) in this approach.

\subsection{MOR for stochastic expansions}

We demonstrate the potential to apply MOR methods for the numerical computation of the unknown coefficient functions in the PC expansions introduced in Section 10.2.4.

\subsubsection{Stochastic Galerkin method}

Let $\mathbf{v}=\left(\mathbf{v}_{1}^{\top}, \ldots, \mathbf{v}_{m}^{\top}\right)^{\top} \in \mathbb{R}^{m n}$ and $\mathbf{w}=\left(w_{1}, \ldots, w_{m}\right)^{\top} \in \mathbb{R}^{m}$. The random-dependent system (10.3) changes into a larger coupled linear dynamical system

$$
\begin{aligned}
\hat{E} \dot{\mathbf{v}}(t) & =\hat{A} \mathbf{v}(t)+\hat{B} \mathbf{u}(t), \\
\mathbf{w}(t) & =\hat{C} \mathbf{v}(t),
\end{aligned}
$$


with constant matrices $\hat{A}, \hat{E} \in \mathbb{R}^{m n \times m n}, \hat{B} \in \mathbb{R}^{m n \times n_{\text {in }}}$, and $\hat{C} \in \mathbb{R}^{m \times m n}$. Initial values $\mathbf{v}\left(t_{0}\right)=\mathbf{v}_{0}$ follow from a truncated PC expansion of the initial condition (10.2). The stochastic Galerkin system (10.18) always features multiple outputs even if the original system (10.3) has a single output. The number of inputs remains the same.

To define the matrices in the coupled system, we introduce an auxiliary matrix and a column vector by

$$
S(\boldsymbol{\mu})=\left(\Phi_{i}(\boldsymbol{\mu}) \Phi_{j}(\boldsymbol{\mu})\right)_{i, j=1, \ldots, m} \in \mathbb{R}^{m \times m} \quad \text { and } \quad \mathbf{s}(\boldsymbol{\mu})=\left(\Phi_{i}(\boldsymbol{\mu})\right)_{i=1, \ldots, m} \in \mathbb{R}^{m}
$$

The matrices follow from the original matrices in (10.3) by probabilistic integrals

$$
\hat{A}=\mathbb{E}[S \otimes A], \quad \hat{B}=\mathbb{E}[\mathbf{s} \otimes B], \quad \hat{C}=\mathbb{E}[S \otimes C], \quad \hat{E}=\mathbb{E}[S \otimes E]
$$

using the Kronecker product $\otimes$, where the expected values (10.6) are calculated componentwise. If the matrices of the system (10.3) represent polynomials of the random variables, then the expected values can often be calculated analytically. Otherwise, numerical quadrature schemes are required to calculate the matrices once.

The linear Galerkin system (10.18) may be unstable, even though the systems (10.3) are asymptotically stable for (strictly) all realizations of the random variables. However, such a loss of stability hardly occurs within problems from the applications. Examples of stability loss are just academic; cf. [37]. Thus we assume that the stochastic Galerkin system (10.18) is asymptotically stable. More details on the stochastic Galerkin method for linear dynamical systems can be found in [31, 32], for example.

The dimension of the stochastic Galerkin system (10.18) is $m n$. This dimensionality becomes huge for large numbers $m$ given by (10.13). Thus the linear stochastic Galerkin system represents an excellent candidate for an MOR. Projection-based MOR operates on the constant matrices $\hat{A}, \hat{B}, \hat{C}, \hat{E}$ like in (10.5). Krylov subspace methods were successfully applied in $[23,47]$. Balanced truncation was used in $[15,33,35]$. The reduction is often efficient such that reduced dimensions $r<m$ are still sufficiently accurate, i. e., the state space dimension is lower than the number of outputs.

\subsubsection{Stochastic collocation techniques}

If the stochastic model is solved approximately using the solutions of the dynamical system (10.3) for a finite number of realizations of the random parameters, then the approach is called a stochastic collocation method. In this context, we use a quadrature rule or a sampling scheme introduced in Section 10.2.3 to compute the unknown coefficient functions (10.15) of the PC expansion.

The original dynamical systems (10.3) may be small- or medium-sized. To make the stochastic collocation method accessible to MOR, we construct a large auxiliary system; see [33, 34]. A construction of this type was also applied to Itô differential 
equations for another purpose in [27]. Given the nodes of a quadrature rule or sampling scheme, the initial value problems

$$
\begin{aligned}
E\left(\boldsymbol{\mu}^{(\ell)}\right) \dot{\mathbf{x}}\left(t, \boldsymbol{\mu}^{(\ell)}\right) & =A\left(\boldsymbol{\mu}^{(\ell)}\right) \mathbf{x}\left(t, \boldsymbol{\mu}^{(\ell)}\right)+B\left(\boldsymbol{\mu}^{(\ell)}\right) \mathbf{u}(t), \quad \mathbf{x}\left(t_{0}\right)=\mathbf{x}_{0}\left(\boldsymbol{\mu}^{(\ell)}\right), \\
y\left(t, \boldsymbol{\mu}^{(\ell)}\right) & =C\left(\boldsymbol{\mu}^{(\ell)}\right) \mathbf{x}\left(t, \boldsymbol{\mu}^{(\ell)}\right)
\end{aligned}
$$

are solved separately for $\ell=1, \ldots, k$. The integrals in (10.15) change into the finite sums

$$
w_{i}(t)=\sum_{\ell=1}^{k} \gamma_{\ell} \Phi_{i}\left(\boldsymbol{\mu}^{(\ell)}\right) y\left(t, \boldsymbol{\mu}^{(\ell)}\right)=\sum_{\ell=1}^{k} \gamma_{\ell} \Phi_{i}\left(\boldsymbol{\mu}^{(\ell)}\right) C\left(\boldsymbol{\mu}^{(\ell)}\right) \mathbf{x}\left(t, \boldsymbol{\mu}^{(\ell)}\right)
$$

for $i=1,2, \ldots, m$.

The systems (10.20) are collected in a single system as done in [33]. Let

$$
\begin{aligned}
\hat{\mathbf{x}}(t) & :=\left(\mathbf{x}\left(t, \boldsymbol{\mu}^{(1)}\right)^{\top}, \ldots, \mathbf{x}\left(t, \boldsymbol{\mu}^{(k)}\right)^{\top}\right)^{\top} \in \mathbb{R}^{k n} \text { and } \\
\mathbf{w}(t) & :=\left(w_{1}(t), \ldots, w_{m}(t)\right)^{\top} \in \mathbb{R}^{m} .
\end{aligned}
$$

The systems (10.20) for $\ell=1, \ldots, k$ together with the outputs $(10.21)$ for $i=1, \ldots, m$ yield the larger weakly coupled system of the form (10.18). This system consists of $k$ separate subsystems (10.20), which are coupled only by supplying of the same input and the definition of the outputs (10.21). Thus the matrices $\hat{A}, \hat{E} \in \mathbb{R}^{k n \times k n}$ are blockdiagonal. More precisely, we have

$$
\hat{G}=\left(\begin{array}{ccc}
G\left(\boldsymbol{\mu}^{(1)}\right) & & \\
& \ddots & \\
& & G\left(\boldsymbol{\mu}^{(k)}\right)
\end{array}\right) \quad \text { for } G \in\{A, E\} \text { and } \quad \hat{B}=\left(\begin{array}{c}
B\left(\boldsymbol{\mu}^{(1)}\right) \\
\vdots \\
B\left(\boldsymbol{\mu}^{(k)}\right)
\end{array}\right) \text {. }
$$

Obviously, the weakly coupled system is asymptotically stable provided that the original systems (10.3) are asymptotically stable for all $\boldsymbol{\mu} \in \mathcal{D}$. Likewise, we define an auxiliary matrix

$$
\widetilde{C}=\left(\begin{array}{ccc}
C\left(\boldsymbol{\mu}^{(1)}\right) & & \\
& \ddots & \\
& & C\left(\boldsymbol{\mu}^{(k)}\right)
\end{array}\right) \in \mathbb{R}^{k \times k n}
$$

The quadrature rule (10.21) determines the output matrix $\hat{C} \in \mathbb{R}^{m \times k n}$ by

$$
\hat{C}=F \widetilde{C} \quad \text { with } F=\left(f_{i \ell}\right) \in \mathbb{R}^{m \times k}, \quad f_{i \ell}=\gamma_{\ell} \Phi_{i}\left(\boldsymbol{\mu}^{(\ell)}\right) .
$$

Again the outputs w of (10.18) yield an approximation (10.16) of the QoI. For large numbers $k$ of nodes or samples, the dimension $k n$ becomes huge. Now we can apply methods of MOR to the weakly coupled system. 


\subsection{Parametric MOR for quadrature and sampling}

We show a potential to compute statistics, where ROMs from pMOR are sampled instead of the full-order models (FOMs). Hence the ROM is used as a surrogate model. This approach is applicable to both linear dynamical systems (10.3) and nonlinear dynamical systems (10.1). The previous works [5, 8] represent surveys on pMOR. In [38], UQ and pMOR have already been combined in the case of linear dynamical systems. A specific pMOR method is presented for general dynamical systems in Chapter 7 of Volume 1 of Model order reduction.

\subsubsection{Application of pMOR}

Now the original parameter-dependent dynamical system is assumed to be highdimensional. The aim of pMOR is to preserve the parameters in a reduction of the systems (10.1) or (10.3). Thus the ROMs are constructed in dependence on the parameters within an offline phase, where the computation work is significant. Whenever an ROM is required for a particular realization of the parameters, a cheap formula is available within an online phase.

In projection-based pMOR, there are mainly two possibilities to determine the projection matrices:

1. Parameter-dependent projections: A priori calculations yield formulas for the projection matrices $V(\boldsymbol{\mu}), W(\boldsymbol{\mu}) \in \mathbb{R}^{n \times r}$, which can be evaluated for any $\boldsymbol{\mu} \in \mathcal{D}$. The reduced matrices become

$$
\begin{aligned}
\bar{A}(\boldsymbol{\mu}) & =W(\boldsymbol{\mu})^{\top} A(\boldsymbol{\mu}) V(\boldsymbol{\mu}), & \bar{B}(\boldsymbol{\mu}) & =W(\boldsymbol{\mu})^{\top} B(\boldsymbol{\mu}), \\
\bar{C}(\boldsymbol{\mu}) & =C(\boldsymbol{\mu}) V(\boldsymbol{\mu}), & & \bar{E}(\boldsymbol{\mu})=W(\boldsymbol{\mu})^{\top} E(\boldsymbol{\mu}) V(\boldsymbol{\mu}),
\end{aligned}
$$

for varying parameters $\boldsymbol{\mu} \in \mathcal{D}$. For example, a local reduced basis is computed for each element in a predetermined finite set of parameters. If the projection matrices are required for a particular $\boldsymbol{\mu} \in \mathcal{D}$, then a kind of interpolation produces $V(\boldsymbol{\mu})$ and $W(\boldsymbol{\mu})$ using neighboring local bases.

2. Constant projections: The information of the whole parameter domain $\mathcal{D}$ or a large finite set of samples is used to construct constant projection matrices $V_{0}, W_{0}$. Thus we have

$$
V(\boldsymbol{\mu})=V_{0} \quad \text { and } \quad W(\boldsymbol{\mu})=W_{0} \quad \text { for all } \boldsymbol{\mu} \in \mathcal{D}
$$

in (10.22). This approach yields global projection matrices, which can be used for any realization of the parameters. However, a larger reduced dimension is often necessary for a sufficiently accurate MOR in comparison to a local construction.

In both cases, the two projection matrices are often identically selected $(W=V)$ and thus just one projection matrix has to be identified. 
In the two variants of projection-based pMOR, the crucial computation work takes place in an offline phase. Often this offline phase consists in evaluations of the FOM for a finite set of parameter values. Such evaluations could also be used in a quadrature method applied to the FOM without an MOR. Thus the critical issue is if the pMOR is able to identify a sufficiently accurate global ROM still with a low computational effort. Otherwise, the FOM could be sampled as well. This problem of certified accuracy in pMOR has also been recognized by [11]. If the error of the pMOR does not exceed the magnitude of the error in the time integration, then the described approach is reasonable.

A stochastic Galerkin method can be applied to a parameter-dependent ROM; see [38]. This approach features both advantages and disadvantages in comparison to the strategy from Section 10.3.1. Alternatively, we apply the approach of quadrature or sampling to the ROMs in this section.

\subsubsection{Computation of global projection matrix}

We demonstrate a technique to determine a global projection matrix from a set of local projection matrices. Alternative strategies can be found in [5, 14, 41]. Let parameter values $\left\{\boldsymbol{\mu}^{(1)}, \ldots, \boldsymbol{\mu}^{(s)}\right\} \subset \mathcal{D}$ be given, which should generate a good representation of all parameters within $\mathcal{D}$. We determine local projection matrices $V\left(\boldsymbol{\mu}^{(j)}\right) \in \mathbb{R}^{n \times r_{j}}$ for $j=1, \ldots, s$ by some MOR technique applied to the dynamical system (10.1) or (10.3). These local projection matrices are not required to be orthogonal. We collect all local bases in a large matrix

$$
\hat{V}=\left(V\left(\boldsymbol{\mu}^{(1)}\right) V\left(\boldsymbol{\mu}^{(2)}\right) \cdots V\left(\boldsymbol{\mu}^{(s)}\right)\right) \in \mathbb{R}^{n \times \hat{r}}
$$

with $\hat{r}=r_{1}+r_{2}+\cdots+r_{s}$ columns, assuming $\hat{r} \ll n$. In [20], just an orthogonalization of a matrix like (10.24) is applied to define the global projection matrix.

We decrease the dimension $\hat{r}$ of the global basis further by an approach also used in [38]. Moreover, this technique removes a (numerical) rank deficiency in the matrix (10.24) if so. The singular value decomposition (SVD) of the matrix (10.24) reads as

$$
\hat{V}=U S Q^{\top}
$$

with orthogonal matrices $U \in \mathbb{R}^{n \times n}, Q \in \mathbb{R}^{\hat{r} \times \hat{r}}$ and a diagonal matrix $S \in \mathbb{R}^{n \times \hat{r}}$ including the nonnegative singular values $\sigma_{1} \geq \sigma_{2} \geq \cdots \geq \sigma_{\hat{r}}$. In our application, just the first $\hat{r}$ singular values and their singular vectors have to be computed, which makes the SVD cheap. The singular vectors are the columns $\mathbf{u}_{1}, \ldots, \mathbf{u}_{\hat{r}}$ of the matrix $U$. Depending on the decay of the singular values, the $r$ dominant singular vectors are entered in the global basis $(r<\hat{r})$

$$
V_{0}=\left(\mathbf{u}_{1}, \mathbf{u}_{2}, \ldots, \mathbf{u}_{r}\right) \in \mathbb{R}^{n \times r}
$$


Thus the global matrix $V$ is already orthogonal $\left(V_{0}^{\top} V_{0}=I_{r}\right)$. Furthermore, we simply employ the Galerkin-type choice $W_{0}=V_{0}$ to define the second projection matrix in (10.23).

If there were no parameter variations in the dynamical system, then the local projection matrices would be identical provided that the same MOR scheme is used. In (10.24), it follows that $V\left(\boldsymbol{\mu}^{(k)}\right)=V\left(\boldsymbol{\mu}^{(0)}\right) \in \mathbb{R}^{n \times r_{0}}$ for all $k=1, \ldots, s$ with any reference parameter $\boldsymbol{\mu}^{(0)} \in \mathcal{D}$. Now let this constant projection matrix be orthogonal. Consequently, the extended matrix (10.24) owns the singular values

$$
\sigma_{i}= \begin{cases}\sqrt{s} & \text { for } i=1, \ldots, r_{0}, \\ 0 & \text { for } i=r_{0}+1, \ldots, s r_{0} .\end{cases}
$$

If a low amount of parameter variation is given in $\mathcal{D}$, then the singular values of (10.24) will be close to the trivial instance (10.27). Thus the deviation of the singular values from the case (10.27) provides a measure of the sensitivity of the problem with respect to the parameter variation.

\subsubsection{Reduced basis methods}

The class of reduced basis methods represents a type of pMOR. In particular, this approach is efficient in the case of stationary solutions of PDEs; cf. [18, 22]. For example, weak formulations of elliptic equations can be tackled.

We consider a general problem

$$
L(\mathbf{x}(\boldsymbol{\mu}), \boldsymbol{\mu})=0
$$

defined by a (differential) operator $L: \mathcal{X} \times \mathcal{D} \rightarrow \mathbb{R}$ on a function space $\mathcal{X}$ with norm $\|\cdot\|_{\mathcal{X}}$. Let a unique solution $\mathbf{x} \in \mathcal{X}$ exist for each $\boldsymbol{\mu} \in \mathcal{D}$. The operator $L$ may be a weak formulation of a PDE. Alternatively, $L$ can identify a time-dependent solution of ODEs like a periodic steady-state response, for example. Moreover, a (spatial) discretization of PDEs yields operators whose solutions represent high-dimensional approximations to the exact solutions of an underlying problem. Typical spatial discretizations are finite element methods and finite difference schemes.

Now we assume that a solution of (10.28) has to be computed many times for different realizations of the parameters. Thus we want to use a surrogate model that generates cheap approximations. In reduced basis methods, a relatively small set of linearly independent solutions is identified, which form the subspace

$$
\mathcal{X}_{r}=\operatorname{span}\left\{\mathbf{x}\left(\boldsymbol{\mu}^{(1)}\right), \ldots, \mathbf{x}\left(\boldsymbol{\mu}^{(r)}\right)\right\}
$$

Given an arbitrary parameter value $\boldsymbol{\mu}$, the associated solution of (10.28) is approximated by a linear combination

$$
\mathbf{x}(\boldsymbol{\mu}) \approx \sum_{j=1}^{r} \alpha_{j}(\boldsymbol{\mu}) \mathbf{x}\left(\boldsymbol{\mu}^{(j)}\right),
$$


where the real coefficients $\alpha_{1}, \ldots, \alpha_{r}$ have to be determined in dependence on the parameter value. Consequently, the operator (10.28) is modified into an approximation

$$
L_{r}\left(\mathbf{x}_{r}^{*}(\boldsymbol{\mu}), \boldsymbol{\mu}\right)=0
$$

with $L_{r}: \mathcal{X}_{r} \times \mathcal{D} \rightarrow \mathbb{R}$, which identifies an approximation in the subspace $\mathcal{X}_{r}$ and thus the required coefficients of (10.30).

Two tasks have to be accomplished in the reduced basis approach:

1. determination of the basis functions in (10.29),

2. construction of the reduced-order operator (10.31) and its efficient numerical solution.

The first task is typically achieved by a greedy algorithm. We approximate the parameter domain $\mathcal{D}$ by a large finite set of samples $\mathcal{D}_{\text {train }} \subset \mathcal{D}$. Let an initial solution $\mathbf{x}\left(\boldsymbol{\mu}^{(1)}\right)$ be given. We compute the subspaces (10.29) recursively via $\mathcal{X}_{j+1}=\mathcal{X}_{j} \cup \operatorname{span}\left\{\mathbf{x}\left(\boldsymbol{\mu}^{j+1}\right)\right\}$ with

$$
\boldsymbol{\mu}^{(j+1)}=\underset{\boldsymbol{\mu} \in \mathcal{D}_{\text {train }}}{\arg \max }\left\|\mathbf{x}(\boldsymbol{\mu})-\mathbf{x}_{j}^{*}(\boldsymbol{\mu})\right\|_{\mathcal{X}}
$$

including the solutions of the operators (10.28) and (10.31). However, the computation of the solutions and the norm of their difference is often too costly in (10.32). Hence we replace the norm by a residual-based estimator $R$. The bounds

$$
c_{1} R(\boldsymbol{\mu}) \leq\left\|\mathbf{x}(\boldsymbol{\mu})-\mathbf{x}_{j}^{*}(\boldsymbol{\mu})\right\|_{\mathcal{X}} \leq c_{2} R(\boldsymbol{\mu}) \quad \text { for } \boldsymbol{\mu} \in \mathcal{D}
$$

with constants $c_{1}, c_{2}>0$ are required for a certified error estimation. The computational effort of an evaluation of the residual-based criterion is low.

The second task consists in the derivation of the approximation (10.31) to the operator (10.28). This construction is problem-dependent. In weak formulations of PDEs, the original function space is just restricted to the low-dimensional subspace (10.29). In the case of linear operators, we compute the involved matrices a priori in the offline phase. The solution of (10.31) becomes cheap in the online phase now. In the case of nonlinear operators, a straightforward approximation still includes the complete nonlinear terms of (10.28). Thus we require cheap approximations of the nonlinearities. The (discrete) empirical interpolation method represents such an approximate construction; see [10] and the references therein.

The efficiency of reduced basis methods can be motivated by the manifold of the parametric solutions

$$
\mathcal{M}=\{\mathbf{x}(\boldsymbol{\mu}): \boldsymbol{\mu} \in \mathcal{D}\} \subset \mathcal{X} .
$$

If the dependence of the PDE solutions on the parameters is (sufficiently) smooth, then the Kolmogorov width of the manifold is small. Consequently, a sufficiently accurate approximation is possible by a low-dimensional subspace. 
Stochastic reduced basis methods were already investigated in [26, 39]. The use of reduced basis methods for UQ of weak formulations of PDEs was presented in detail by [11]. The reduced basis approach was proven to be efficient for stationary problems in a spatial domain. Also parabolic equations which depend on time as well as space can be treated by these methods; see $[11,30]$. However, the applicability to transient problems like our dynamical systems (10.1) and (10.3) still has to be examined. We think about dynamical systems which do not result from a spatial discretization of a PDE. In this case, the function space $\mathcal{X}$ may represent the periodic steady-state response in a time interval $[0, T]$, since periodic solutions satisfy a boundary value problem. The efficiency is still undecided for initial value problems. If just the solution's value in $\mathbb{R}^{n}$ at a final time $t=T$ represents the QoI, then reduced basis methods are unnecessarily complex for this task.

\subsection{Numerical examples}

We demonstrate the application of the approaches from Section 10.3 and Section 10.4 now.

\subsubsection{MOR for stochastic expansions}

Figure 10.3 depicts the diagram of a band pass filter. The mathematical modeling yields an explicit system of ODEs with dimension $n=10$ for five node voltages and five branch currents. Physical parameters are included by five capacitances, five inductances, and four resistances ( $p=14)$. A single input voltage is supplied, whereas a single output voltage drops at a load resistance. The Bode plot of the linear dynamical system is shown for a constant choice of the parameters by Figure 10.4. We recognize that there is just a small frequency interval around $\omega=1$, where the magnitude of oscillations remains the same, while other frequencies are damped strongly.

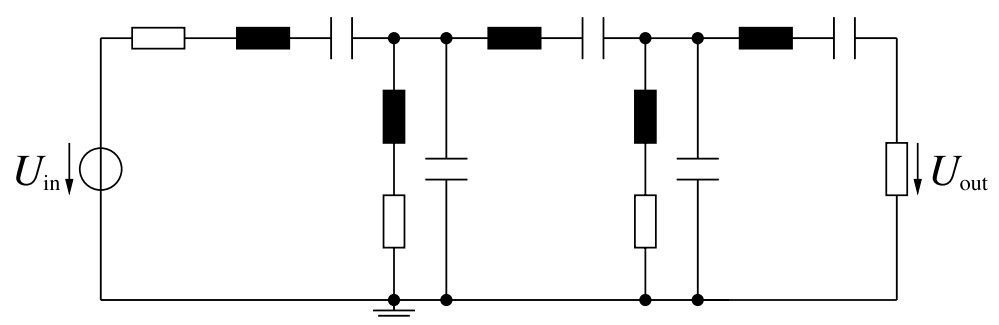

Figure 10.3: Electric circuit of a band pass filter. 

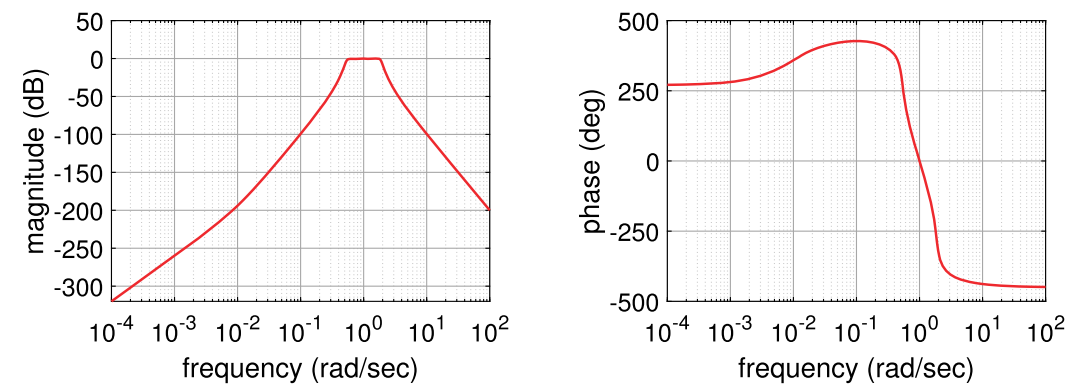

Figure 10.4: Bode plot of band pass filter for deterministic parameters.

We replace all physical parameters by independent random variables with uniform probability distributions, which vary $20 \%$ around their mean values given by the constant choice of parameters from above. Hence the PC expansion (10.14) involves the multivariate Legendre polynomials. In the truncated PC expansion (10.16), we include all basis polynomials up to total degree $d=3$, which implies $m=680$ basis functions due to (10.13).

Now the two approaches from Section 10.3 are examined. On the one hand, we arrange the stochastic Galerkin system of dimension $m n=6800$ as in Section 10.3.1. The system matrices (10.19) are computed by a sparse grid quadrature of level 3 based on the Clenshaw-Curtis rule, where $k=4117$ nodes arise. On the other hand, we generate a stochastic collocation system as in Section 10.3.2, with a sparse grid quadrature of level 2 of the same type with $k=421$ nodes. The dimension of this weakly coupled system is $k n=4210$. Both systems feature a single input and $m$ outputs. The outputs reproduce the expected value as well as the variance of the output voltage via (10.17).

We perform an MOR of both linear dynamical systems using the balanced truncation technique; see [2]. A direct linear algebra algorithm yields the Cholesky factors of the Gramian matrices. An SVD produces the Hankel singular values in each approach, which are shown in Figure 10.5. The singular values exhibit a similar rate of decay in both linear dynamical systems. We expect a high potential for an MOR due to the fast decay. The singular values and singular vectors allow for the construction of ROMs with any dimension.

We also perform a transient simulation to compare the accuracy of the stochastic expansion methods and their ROMs. The input voltage is chosen as the chirp signal

$$
u(t)=\sin \left(2 \pi t^{2}\right)
$$

The output voltage represents the random QoI. The time interval $[0,100]$ is considered with initial values (10.2) identical to zero. In the time integration, we use the trapezoidal rule with constant step size $\Delta t=0.005$ in all cases. A reference solution of the expected value as well as the variance is computed using directly the sparse grid quadrature of level 3 with $k=4117$ nodes (without projection to a PC expansion). 


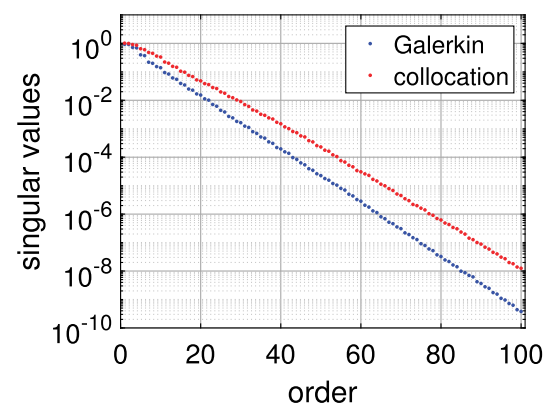

Figure 10.5: Dominating Hankel singular values of linear dynamical systems from the stochastic Galerkin method and the stochastic collocation technique in the band pass filter example.

Hence the error of the time integration becomes negligible in comparison to the error of the stochastic discretizations and the error of an MOR for moderate reduced dimensions. Figure 10.6 shows the approximations for the expected value as well as the standard deviation in the FOMs. The approximations of the expected value coincide in all techniques. The approximations of the standard deviation agree for the stochastic Galerkin method. The stochastic collocation yields a slightly different approximation, which still captures the main dynamics. Now we consider the ROMs in the stochastic Galerkin method and the stochastic collocation. Table 10.1 illustrates the differences of the expected value as well as the variance with respect to the FOM solution for varying reduced dimensions. Obviously, the differences diminish for increasing dimensions, which confirms the quality of the used MOR.
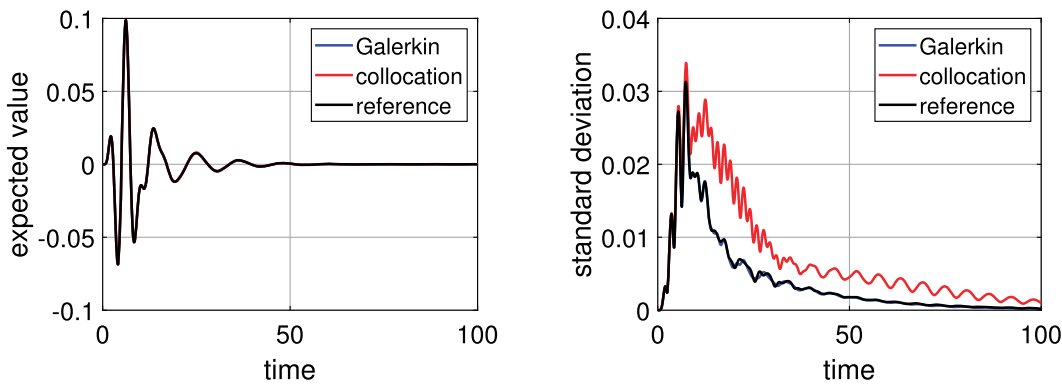

Figure 10.6: Transient simulation of expected value (left) and standard deviation (right) for random output voltage from the stochastic Galerkin approach, the stochastic collocation scheme, and reference solution.

In this example, we reproduced the expected value and the variance. Nevertheless, more sophisticated quantities can be derived from the PC approximation (10.16) using the coefficient functions. 
Table 10.1: Maximum differences (rounded to one digit) in moments between FOM and ROM for the stochastic Galerkin method and the stochastic collocation technique.

\begin{tabular}{llrrr}
\hline Reduced dimension & $\mathbf{1 0}$ & $\mathbf{2 0}$ & $\mathbf{3 0}$ \\
\hline Galerkin & Expected value & $8 \cdot 10^{-3}$ & $7 \cdot 10^{-4}$ & $2 \cdot 10^{-4}$ \\
& Variance & $1 \cdot 10^{-4}$ & $2 \cdot 10^{-5}$ & $1 \cdot 10^{-6}$ \\
Collocation & Expected value & $2 \cdot 10^{-2}$ & $3 \cdot 10^{-3}$ & $4 \cdot 10^{-4}$ \\
& Variance & $3 \cdot 10^{-4}$ & $2 \cdot 10^{-5}$ & $7 \cdot 10^{-6}$ \\
\hline
\end{tabular}

\subsubsection{PMOR for statistics}

The anemometer system represents a benchmark in MOR; see [24, 25]. The convectiondiffusion PDE

$$
\rho_{\mathrm{fl}} c \frac{\partial T}{\partial t}=\nabla \cdot(\kappa \nabla T)-\rho_{\mathrm{fl}} c(\mathbf{v} \cdot \nabla T)+\dot{q}
$$

models the time evolution of the temperature $T$ with fluid density $\rho_{\mathrm{fl}}$, thermal conductivity $\kappa$, specific heat $c$, and the velocity profile $\mathbf{v}$. The heat flow $\dot{q}$ becomes the input. The output is defined as the temperature difference between two sensors. We obtain a rough estimate of the flow velocity $v$ (as a part of $\mathbf{v}$ ) by this difference. A finite element method performs a spatial discretization, which generates an implicit system of linear ODEs (10.3) with dimension $n=29008$ and single-input-single-output. We arrange a constant fluid density $\rho_{\mathrm{fl}}=1$. The system still depends on the three positive parameters $\mu_{1}=c, \mu_{2}=v, \mu_{3}=\kappa$. Figure 10.7 depicts the Bode plot of the linear dynamical system in the case of deterministic parameters $\mu_{i}=1$ for $i=1,2,3$.
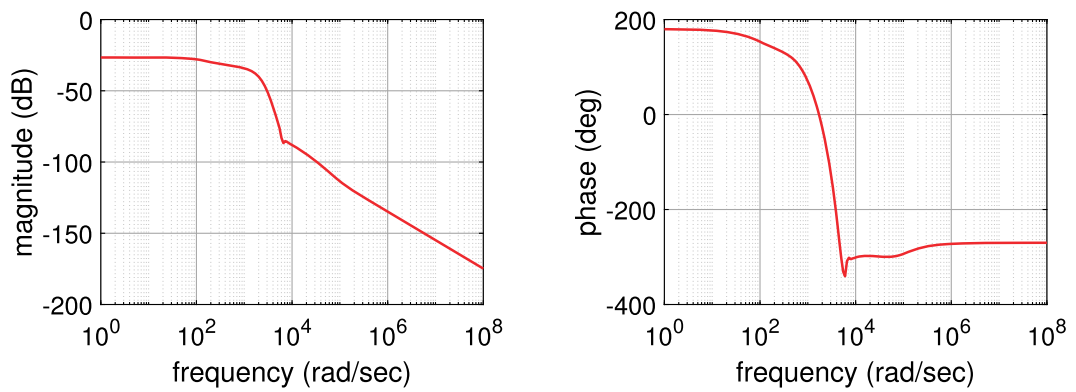

Figure 10.7: Bode plot of anemometer model for deterministic parameters.

In the stochastic modeling, we choose independent beta distributions for each parameter. Given a single beta-distributed random variable $\mu \in[-1,1]$, the probability density function reads as

$$
\rho(\mu)=C(\alpha, \beta)(1-\mu)^{\alpha}(1+\mu)^{\beta},
$$


with exponents $\alpha, \beta>-1$ and a constant $C(\alpha, \beta)>0$ for normalization. A bijective linear transformation maps the interval $[-1,1]$ to any interval $\left[\mu_{\min }, \mu_{\max }\right]$. We consider two choices of the parameter domain $\mathcal{D}$ :

(i) small variation: $\mu_{1} \in[0.9,1.0], \mu_{2} \in[1.0,1.1], \mu_{3} \in[1.0,1.1]$,

(ii) large variation: $\mu_{1} \in[0.5,1.0], \mu_{2} \in[0.7,1.2], \mu_{3} \in[1.0,1.5]$.

Furthermore, we select the exponents $\alpha_{i}=1$ and $\beta_{i}=3$ for all $i=1,2,3$.

In a pMOR, we use the technique from Section 10.4.2. We choose all vertices of the cube $\mathcal{D}$ for the computation of local reduced bases. Hence $s=8$ parameter samples are involved. The one-sided Arnoldi method (see [2]) represents a moment-matching method, where a single expansion point $\theta \in \mathbb{C}$ is applied in the frequency domain. We employ the real expansion point $\theta=10^{4}$ for each parameter sample, which causes real-valued results. A local orthonormal basis of dimension $r_{j}=25$ is generated for each parameter sample $j=1, \ldots, s$. The extended matrix (10.24) consists of $\hat{r}=200$ columns. We compute its SVD (10.25) in the two cases (i) and (ii) of the parameter domains. The singular values are depicted in Figure 10.8. As expected, the singular values behave similar to the limit (10.27) of vanishing parameter dependence in the case (i) of small variations. The rate of decay becomes slower in the case (ii) of larger variations. Now a global reduced basis (10.26) can be arranged for any dimension $r \leq \hat{r}$, where the singular vectors associated to the dominant singular values are included.

(i)

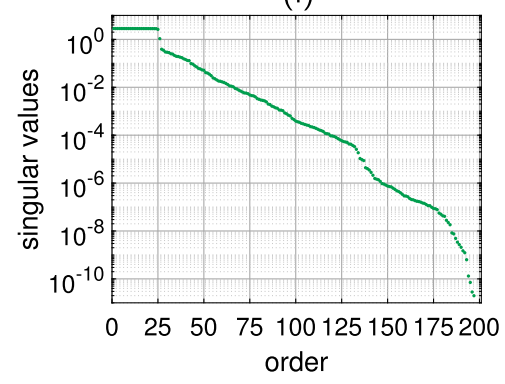

(ii)

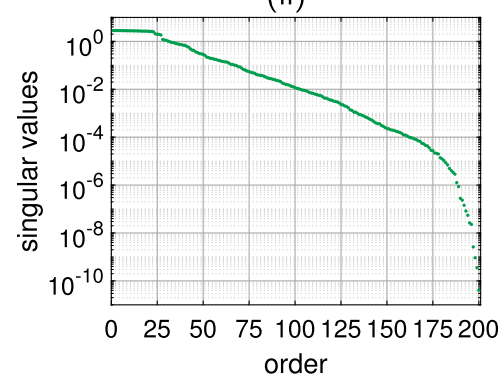

Figure 10.8: Singular values of extended matrix (10.24) in PMOR for the two choices (i) and (ii) of the parameter domain in the anemometer example.

We perform a transient simulation for a comparison of the FOM and the ROM in the case (ii). Using the time interval [0,0.01], the harmonic oscillation

$$
u(t)=A \sin \left(\frac{2 \pi}{T} t\right)
$$

is supplied as input with period $T=10^{-4}$ and amplitude $A=10^{4}$. Initial values are identical to zero. The trapezoidal rule performs a time integration with constant step size $\Delta t=\frac{T}{20}$. Our aim is to compute statistics of the random process induced by 
the single output. We use the Gauss-Jacobi quadrature on a tensor product grid with $k=4^{3}=64$ nodes. Further tests indicate that this quadrature scheme is sufficiently accurate. In each node, the initial value problem is solved numerically for both the FOM and an ROM.

Firstly, we select the dimension $r=75$ of the global basis in the ROM. Figure 10.9 illustrates the expected value as well as the standard deviation for both FOM and ROM. The approximation of the expected value is appropriate, whereas the standard deviation includes incorrect oscillations in the ROM. Secondly, we arrange the reduced dimension $r=125$. Figure 10.10 shows the expected value and the standard deviation again. Now the solution from the ROM represents a good approximation to both statistical quantities. Furthermore, the skewness and the kurtosis (see (10.8)), which relate to the third moment and the fourth moment, respectively, are displayed in Figure 10.11. Although the approximations of the higher moments are less accurate in the MOR, the dynamics as well as the magnitude of these statistics are captured correctly.
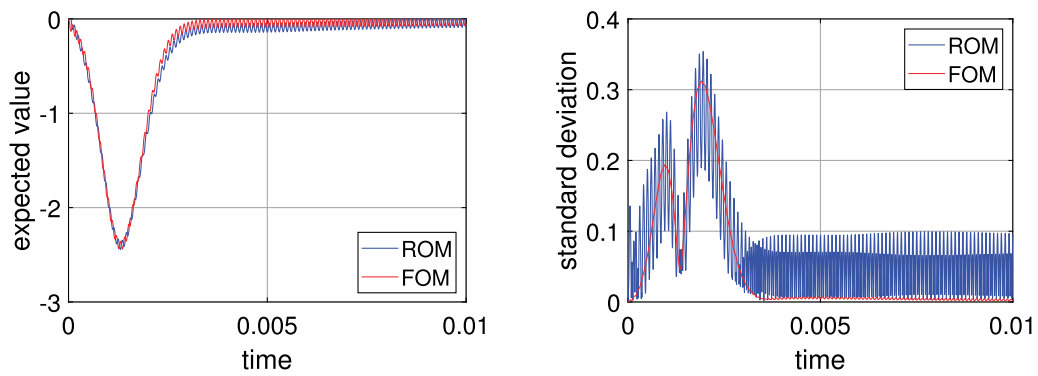

Figure 10.9: Expected value (left) and standard deviation (right) of random output in anemometer system obtained by ROM of dimension $r=75$.
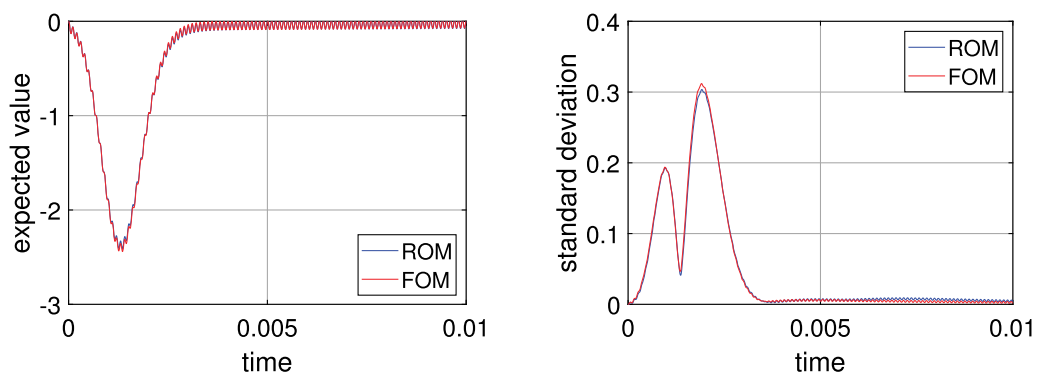

Figure 10.10: Expected value (left) and standard deviation (right) of random output in the anemometer system obtained by the ROM of dimension $r=125$.

We note that a global projection matrix computed for a parameter domain $\mathcal{D}$ can be reused for any probability distribution in $\mathcal{D}$. For example, different exponents may be chosen in the beta distributions (10.34). 

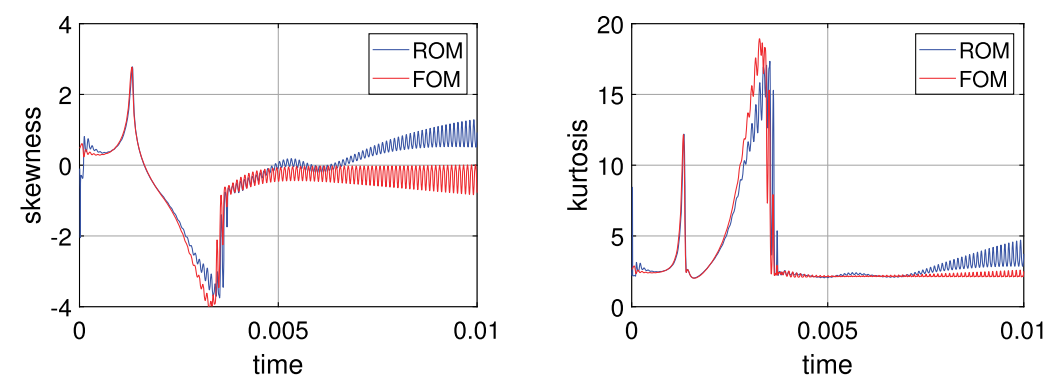

Figure 10.11: Skewness (left) and kurtosis (right) of random output in the anemometer system obtained by the ROM of dimension $r=125$.

\subsection{Conclusions and outlook}

We discussed two approaches for MOR of dynamical systems including random parameters to model uncertainties. On the one hand, the stochastic Galerkin method and the stochastic collocation technique produce high-dimensional deterministic dynamical systems, which can be reduced by traditional MOR algorithms. On the other hand, pMOR is applied, where an ROM is sampled instead of the FOM within a quadrature scheme or (quasi-)Monte Carlo method.

In the second approach, the computational effort for the construction of the parametric ROM is critical. If this effort becomes too large, then a quadrature scheme applied to the FOM may yield results of the same quality with lower computation work. Hence we require efficient pMOR methods, where error bounds or error estimates are available to decide the quality of an ROM. More precisely, this error should be in the magnitude of the error in a time integration to accept the results.

As usual, MOR of nonlinear dynamical systems is challenging also in the field of UQ. However, the stochastic Galerkin method is often less efficient in the case of nonlinear dynamical systems, since some probabilistic integrals cannot be evaluated analytically. Thus sampling methods and collocation schemes are preferred. One should check if a given nonlinear dynamical system can be converted into a quadratic-bilinear system, either equivalently or approximately. Consequently, efficient MOR methods are available for QB systems. Research on MOR and parametric MOR still continues for nonlinear dynamical systems.

\section{Bibliography}

[1] M. I. Ahmad, P. Benner, and L. Feng, Interpolatory model reduction for quadratic-bilinear systems using error estimators, Eng. Comput., 36 (2019), 25-44.

[2] A. Antoulas, Approximation of Large-Scale Dynamical Systems, SIAM Publications, 2005. 
[3] F. Augustin, A. Gilg, M. Paffrath, P. Rentrop, and U. Wever, Polynomial chaos for the approximation of uncertainties: chances and limits, Eur. J. Appl. Math., 19 (2008), 149-190.

[4] P. Benner, P. K. Goyal, and S. Gugercin, $\mathrm{H}_{2}$-quasi-optimal model order reduction for quadratic-bilinear control systems, SIAM J. Matrix Anal. Appl., 39 (2018), 983-1032.

[5] P. Benner, S. Gugercin, and K. Willcox, A survey of projection-based model order reduction methods for parametric dynamical systems, SIAM Rev., 57 (2015), 483-531.

[6] P. Benner, M. Hinze, and E. J. W. ter Maten (eds.), Model Reduction for Circuit Simulation, Lect. Notes in Electr. Engng., vol. 74, Springer, 2011.

[7] P. Benner, V. Mehrmann, and D. C. Sorensen (eds.), Dimension Reduction of Large-Scale Systems, Lect. Notes Comput. Sci. Engin., vol. 45, Springer, 2005.

[8] P. Benner, M. Ohlberger, A. Patera, G. Rozza, and K. Urban (eds.), Model Reduction of Parameterized Systems, MS\&A, vol. 17, Springer, 2017.

[9] P. Benner and T. Stykel, Model order reduction for differential-algebraic equations: a survey, in A. Ilchmann, T. Reis (eds.), Surveys in Differential-Algebraic Equations IV, pp.107-160, Springer, 2017.

[10] S. Chaturantabut and D. C. Sorensen, Nonlinear model reduction via discrete empirical interpolation, SIAM J. Sci. Comput., 32 (2010), 2737-2764.

[11] P. Chen and Ch. Schwab, Model order reduction methods in computational uncertainty quantification, in R. Ghanem, D. Higdon, H. Owhadi (eds.), Handbook of Uncertainty Quantification, pp.936-990, Springer, 2017.

[12] O. G. Ernst, A. Mugler, H. J. Starkloff, and E. Ullmann, On the convergence of generalized polynomial chaos expansions, ESAIM: Math. Model. Numer. Anal., 46 (2012), 317-339.

[13] L. Feng, Y. Yue, N. Banagaaya, P. Meuris, W. Schoenmaker, and P. Benner, Parametric modeling and model order reduction for (electro-)thermal analysis of nanoelectronic structures, J. Math. Ind., 6 (2016), 10.

[14] J. Fernandez Villena and L. M. Silveira, Multi-dimensional automatic sampling schemes for multi-point modeling methodologies, IEEE Trans. CAD Integr. Circuits Syst., 30 (2011), 1141-1151.

[15] F. D. Freitas, R. Pulch, and J. Rommes, Fast and accurate model order reduction for spectral methods in uncertainty quantification, Int. J. Uncertain. Quantificat., 6 (2016), 271-286.

[16] R. Freund, Model reduction methods based on Krylov subspaces, Acta Numer., 12 (2003), 267-319.

[17] T. Gerstner and M. Griebel, Numerical integration using sparse grids, Numer. Algorithms, 18 (1998), 209-232.

[18] J. S. Hesthaven, B. Stamm, and S. Zhang, Efficient greedy algorithms for high-dimensional parameter spaces with applications to empirical interpolation and reduced basis methods, ESAIM, Math. Model. Numer. Anal., 48 (2011), 259-283.

[19] J. Li and D. Xiu, Evaluation of failure probability via surrogate models, J. Comput. Phys., 229 (2010), 8966-8980.

[20] P. Li, F. Liu, X. Li, L. Pileggi, and S. Nassif, Modeling interconnect variability using efficient parametric model order reduction, in Proc. of Design Automation and Test in Europe Conference (DATE), pp. 958-963, 2005.

[21] P. Manfredi, D. Vande Ginste, D. De Zutter, and F. G. Canavero, Stochastic modelling of nonlinear circuits via SPICE-compatible spectral equivalents, IEEE Trans. Circuits Syst. I, Regul. Pap., 61 (2014), 2057-2065.

[22] I. Martini, B. Haasdonk, and G. Rozza, Certified reduced basis approximation for the coupling of viscous and inviscid parameterized flow models, J. Sci. Comput., 74 (2018), 197-219. 
[23] N. Mi, S. X.-D. Tan, P. Liu, J. Cui, Y. Cai, and X. Hong, Stochastic extended Krylov subspace method for variational analysis of on-chip power grid networks, in Proc. ICCAD, pp. 48-53, 2007.

[24] Ch. Moosmann and A. Greiner, Convective thermal flow problems, in P. Benner, V. Mehrmann, D. C. Sorensen (eds.), Dimension Reduction of Large-Scale Systems, Lect. Notes Comput. Sci. Engin., vol. 45, pp. 341-343, Springer, 2005.

[25] “MOR Wiki,” online document, https://morwiki.mpi-magdeburg.mpg.de/morwiki, cited Sep 2, 2019.

[26] P. B. Nair and A. J. Keane, Stochastic reduced basis methods, AIAA J., 40 (2002), 1653-1664.

[27] M. Navarro Jimenez, O. P. Le Maitre, and O. M. Knio, Nonintrusive polynomial chaos expansions for sensitivity analysis in stochastic differential equations, SIAM/ASA J. Uncertain. Quantificat., 5 (2017), 378-402.

[28] H. Niederreiter, Random Number Generation and Quasi-Monte Carlo Methods, SIAM, New York, 1992.

[29] F. Nobile, R. Tempone, and C. G. Webster, A sparse grid stochastic collocation method for partial differential equations with random input data, SIAM J. Numer. Anal., 46 (2008), 2309-2345.

[30] M. Ohlberger and S. Rave, Reduced basis methods: success, limitations and future challenges, in Proceedings of the Conference Algoritmy, pp. 1-12, 2016.

[31] R. Pulch, Polynomial chaos for linear differential algebraic equations with random parameters, Int. J. Uncertain. Quantificat., 1 (2011), 223-240.

[32] R. Pulch, Stochastic collocation and stochastic Galerkin methods for linear differential algebraic equations, J. Comput. Appl. Math., 262 (2014), 281-291.

[33] R. Pulch, Model order reduction for stochastic expansions of electric circuits, in A. Bartel, $M$. Clemens, M. Günther, E. J. W. ter Maten (eds.), Scientific Computing in Electrical Engineering SCEE 2014, Mathematics in Industry, vol. 23, pp. 223-232, Springer, 2016.

[34] R. Pulch, A Hankel norm for quadrature rules solving random linear dynamical systems, J. Comput. Appl. Math., 316 (2017), 307-318.

[35] R. Pulch, Model order reduction and low-dimensional representations for random linear dynamical systems, Math. Comput. Simul., 144 (2018), 1-20.

[36] R. Pulch, Model order reduction for random nonlinear dynamical systems and low-dimensional representations for their quantities of interest, Math. Comput. Simul., 166 (2019), 76-92.

[37] R. Pulch and F. Augustin, Stability preservation in stochastic Galerkin projections of dynamical systems, SIAM/ASA J. Uncertain. Quantificat., 7 (2019), 634-651.

[38] R. Pulch and E. J. W. ter Maten, Stochastic Galerkin methods and model order reduction for linear dynamical systems, Int. J. Uncertain. Quantificat., 5 (2015), 255-273.

[39] S. K. Sachdeva, P. B. Nair, and A. J. Keane, Hybridization of stochastic reduced basis methods with polynomial chaos expansions, Probab. Eng. Mech., 21 (2006), 182-192.

[40] W. H. A. Schilders, M. A. van der Vorst, and J. Rommes (eds.), Model Order Reduction: Theory, Research Aspects and Applications, Mathematics in Industry, vol. 13, Springer, 2008.

[41] T. Soll and R. Pulch, Sample selection based on sensitivity analysis in parameterized model order reduction, J. Comput. Appl. Math., 316 (2017), 271-286.

[42] B. Sonday, R. Berry, B. Debusschere, and H. Najm, Eigenvalues of the Jacobian of a Galerkin-projected uncertain ODE system, SIAM J. Sci. Comput., 33 (2011), 1212-1233.

[43] A. Stroud, Approximative Calculation of Multiple Integrals, Prentice-Hall, Inc., 1971.

[44] T. J. Sullivan, Introduction to Uncertainty Quantification, Springer, 2015.

[45] D. Xiu and G. E. Karniadakis, The Wiener-Askey polynomial chaos for stochastic differential equations, SIAM J. Sci. Comput., 24 (2002), 619-644. 
[46] D. Xiu, Numerical Methods for Stochastic Computations: A Spectral Method Approach, Princeton University Press, 2010.

[47] Y. Zou, Y. Cai, Q. Zhou, X. Hong, S. X.-D. Tan, and L. Kang, Practical implementation of the stochastic parameterized model order reduction via Hermite polynomial chaos, in Proc. ASP-DAC, pp. 367-372, 2007. 